\title{
A Model Reference Tracking based on an Active Fault Tolerant Control for LPV Systems
}

\author{
Bouali Rabaoui, Mickael Rodrigues, Habib Hamdi, Naceur BenhadjBraiek \\ Advanced Systems Laboratory, Tunisian Polytechnic School, University of Carthage, Tunisia \\ Laboratory of Automatic and Process Control, LAGEP,University of Lyon, France \\ mickael .rodrigues@cran .uhp-nancy .fr, rabaaouibouali@gmail.com
}

\begin{abstract}
This work deals with the problem of a model reference tracking based on the design of an Active Fault Tolerant Control (AFTC) for linear parameter varying (LPV) systems affected by actuator faults and unknown inputs. LPV systems are described by a polytopic representation with measurable gain scheduling functions. The main contribution is to design an Active Fault Tolerant Controller whose control law is described by an adaptive Proportional Integral (PI) structure. This one requires three types of on-line informations which are: reference outputs, measured real outputs and the fault estimation provided respectively by a model reference, sensors and an Adaptive Polytopic Observer (APO). These informations are used to reconfigure the designed controller which is able to compensate the fault effects and to make the closed loop system able to track reference outputs in spite of the presence of actuator faults and disturbances. The controller and the observer gains are obtained by solving a set of linear matrices inequalities (LMIs). Performances of the proposed method are compared to an other previous method in order to underline the relevant results.
\end{abstract}

Keywords: LPV system, Adaptive Polytopic Observer, model reference tracking control, active fault tolerant control, LMIs.

\section{Introduction and context}

The technologies of modern systems have become more and more sophisticated, which increase the need for the stability, the safety and the reliability. These systems might be malfunctional due to faults that can affect them through their actuators, sensors or components. So, it is necessary to design control systems, as Fault Tolerant Control (FTC), in order to cancel the fault effects and to stabilize the system in spite of these malfunctions.

A fault tolerant controller has the ability to compensate the fault by adjusting some parameters to make the overall closed-loop system stable despite faults and disturbance.

In general, the Fault Tolerant Control laws can be classified into two types: Passive FTC (PFTC) and Active FTC (AFTC). In PFTC systems, controllers are fixed and are designed to be robust against a class of presumed faults which is known as a very conservative approach. In contrast to PFTC, AFTC systems react to the system component failures actively by reconfiguring control actions so that the stability and acceptable performance of the entire system can be maintained [19].

For around two decades, the researchers have been interested in developping new FTC methods. In [11], fault diagnosis and fault tolerant control methods with their application to real plants have been developed. The authors in [8] have been proposed a FTC strategy for LPV systems in the case of actuator faults based on using Unknown Input Observer (UIO). In [13], the authors have proposed an actuator fault estimation scheme based on an Adaptive Polytopic Observer (APO) for LPV descriptor systems in the presence of constant or time varying actuator faults. 
The authors in [12] have extended the results published in [13]. Indeed, they have designed an APO in the objective to study the FTC problem for the same class of systems. Based on the informations provided by the APO, a new state feedback control has been developed so as to compensate the effects of constant or time varying actuator faults. The proposed control law therein has allowed the system to be stable but this one has not been able to have a good accuracy.

The development of state, parameter and fault estimation techniques have appeared in [14] and [18] where the authors have used a switched LPV observer and an adaptive observer respectively. The authors in [15] have designed a LPV state observer and state-feedback controller for a Twin Rotor MIMO System (TRMS) described by quasi-LPV model. The proposed technique has used an LPV pole placement method based on LMI regions.

In [10], an adaptive observer has been designed for uncertain multimodels affected by actuator faults and subject to unknown model parameter variations with a FTC approach. Other works as [1] and [2] concerned the Fault Tolerant Tracking Control (FTTC) for uncertain T-S models with unmeasurable premise variables and affected respectively by actuator and sensor faults and only sensor faults.

A FTC strategy using virtual actuators and sensors for LPV systems has been proposed in [16], where an active FTC strategy has been developed in order to reconfigure the virtual actuator/sensor on-line taking into account faults and operating point changes. In [17], the authors have proposed a fault-hiding approach in order to solve the problem of fault tolerant control for a four-wheeled omnidirectional mobile robot. Moreover, a switching LPV virtual actuator has been added to the control loop so as to adapt the faulty plant to the nominal switching LPV controller.

Today, the standard Proportional Integral (PI) and Proportional Integral Derivative (PID) controllers are the most used controllers in the industry. They are characterized by their simplicity and easy adaptation to the controlled system and allow to control a large number of physical quantities in various fields.

The design of FTC systems through PID controller have attracted the attention of several researchers who have interested in developing new techniques in order to tolerate faults by reconfiguring controllers or tuning their gains. Among them, the authors in [6] have proposed an intelligent-based FTC scheme with an evolutionary programming based self-tuning PID fault tolerant controller in order to solve the fault tolerant tracking control problem for unknown nonlinear multi-inputs-multi-outputs (MIMO) systems. The authors in [4] have discussed the problem of PID-FTC system design for linear systems with state and static output feedback. In [9], a FTC approach using an adaptive PID sliding-mode controller is developed for a full scale vehicle dynamic model with an active suspension system in the presence of uncertainties and actuator faults. In fact, the proposed control laws therein, have only standard structures without adding any term for the faults compensation. So, more recently, some research works have appeared in the literature, but it still lacks the on-line faults compensation through adaptive PI or PID controllers especially for LPV systems.

The main goal of the actual paper is to extend existing results so as to improve the accuracy of system outputs. For this we use an adaptive PI control law instead of a state feedback control law as in [1] and [12]. Contrary to [4], [6] and [9] which has proposed a PID controller with a standard control law structure, the proposed control law here is adapted such that becomes able to compensate perfectly the actuator fault effects.

In this paper, a new method based on an adaptive Proportional Integral (PI) control is proposed to construct an Active Fault Tolerant Control (AFTC). To achieve the controller reconfiguration, an Adaptive Polytopic Observer (APO) is used as a Fault Detection and Diagnosis (FDD) module. The proposed approach consists in designing an Active Fault Tolerant Controller which is able to track a model reference in spite of actuator faults and disturbances. Its main goal is to ensure the stability of the closed-loop LPV system with measurable gain scheduling functions. 
This paper is organized as follows. The structure of the LPV system with measurable gain scheduling functions is represented and the problem statement of the paper is formulated by introducing the proposed scheme in section II. The section III is dedicated to design both the controller based on an AFTC and the Fault Detection and Diagnosis module based on an Adaptive Polytopic Observer. Finally, the section IV presents a comparison of two methods on a two-tank process, and the section $\mathrm{V}$ concludes the paper.

Notation: Throughout this paper, I denotes an identity matrix of appropriate dimension.

\section{Problem statement}

Let consider a continuous time LPV system affected by additive actuator faults and disturbances and modeled by the following state space representation [3]:

$$
\left\{\begin{array}{l}
\dot{x}(t)=A(\theta(t)) x(t)+B(\theta(t))\left(u_{f}(t)+f(t)\right)+R(\theta(t)) d(t) \\
y(t)=C x(t)
\end{array}\right.
$$

where $x(t) \in \Re^{n}$ is the state vector, $u_{f}(t) \in \Re^{p}$ is the control input vector, $y(t) \in \Re^{m}$ is the measured output vector, $f(t) \in \Re^{p}$ denotes the actuator fault vector and $d(t) \in \Re^{q}$ represents the unknown disturbance vector. $C$ is a constant matrix but $A(\theta(t)), B(\theta(t))$ and $R(\theta(t))$ are continuous functions which depend on time varying parameter vector $\theta(t) \in \Re^{l}$. This vector is bounded and lies into a hypercube such that:

$$
\theta(t) \in \Im=\left\{\theta: \theta_{\min }(t) \leq \theta(t) \leq \theta_{\max }(t) ; \forall t \geq 0\right\}
$$

By assuming an affine dependance of the parameter vector $\theta(t)$ [12], the system (1) can be described by a polytopic form, where it can be transformed into a convex combination of the vertices of $\Im$ such that:

$$
\left\{\begin{array}{l}
\dot{x}(t)=\sum_{i=1}^{h} \rho_{i}(\theta(t))\left[A_{i} x(t)+B_{i}\left(u_{f}(t)+f(t)\right)+R_{i} d(t)\right] \\
y(t)=C x(t)
\end{array}\right.
$$

where $\rho(\theta(t))$ vary into the convex set $\Omega$.

$$
\Omega=\left\{\rho(\theta(t)) \in \Re^{h}, \rho(\theta(t))=\left[\rho_{1}(\theta(t)), \ldots, \rho_{h}(\theta(t))\right]^{T} ; \rho_{i}(\theta(t)) \geq 0 \text { and } \sum_{i=1}^{h} \rho_{i}(\theta(t))=1\right\}
$$

The matrices $A_{i} \in \Re^{n \times n}, B_{i} \in \Re^{n \times p}, R_{i} \in \Re^{n \times q}$ and $C \in \Re^{m \times n}$ are time invariant for the $i^{t h}$ model represented by a linear form. They characterize the summits $\Im_{i}$ of the polytope which are defined such that $\Delta_{i}=\left[\begin{array}{llll}A_{i} & B_{i} & R_{i} & C\end{array}\right], \forall i \in\left[\begin{array}{lll}1, & \ldots, & h\end{array}\right]$ where $h=2^{l}$.

Generally, the LTI models, which compose the polytopic representation of the LPV system, are blended through on-line measurable scheduling functions that depend on the input, the output of the system, or an external parameter [7]. 


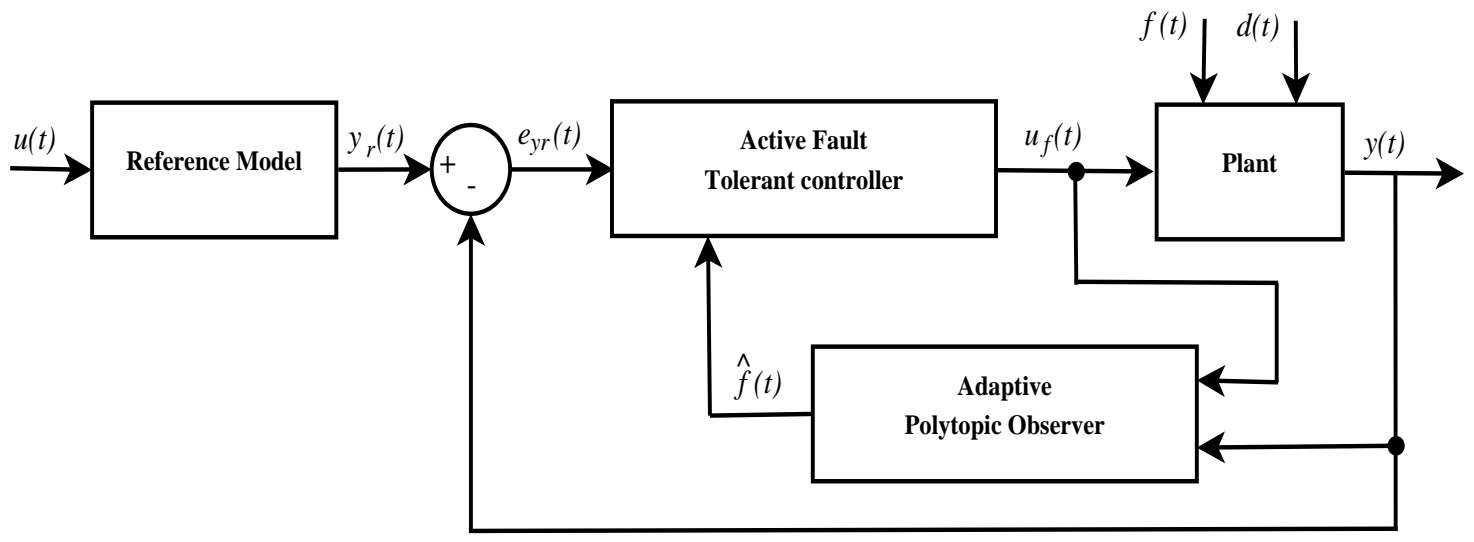

Figure 1: Model reference tracking control Scheme

The main objective of this work is to develop a model reference tracking based on an Active Fault Tolerant Control method. The proposed methodology needs the use of an Adaptive Polytopic Observer (APO) in order to synthesise a feedback control law. The proposed method aims to ensure the asymptotical convergence of the real output to the referential output despite the presence of actuator faults and unknown inputs. The proposed model reference tracking control scheme is illustrated by the figure 1. In fact, the proposed scheme is inspired from the work of (Aouaouda et al.) in [1] which treated the tracking problem of uncertain T-S fuzzy continuous systems with unmeasurable premise variables and affected by unknown inputs. A Proportional Integral (PI) fuzzy observer is used to estimate both constant faults and faulty states so as to synthesize an FTC law.

Let us consider a reference polytopic LPV model given by:

$$
\left\{\begin{array}{l}
\dot{x}_{r}(t)=\sum_{i=1}^{h} \rho_{i}(\theta(t))\left(A_{i} x_{r}(t)+B_{i} u(t)\right) \\
y_{r}(t)=C x_{r}(t)
\end{array}\right.
$$

where $x_{r}(t) \in \Re^{n}, u(t) \in \Re^{p}$ and $y_{r}(t) \in \Re^{m}$ are respectively the state, the control input and the measured output vectors of the model reference.

According to [13], the Adaptive Polytopic Observer (APO) is described by the following structure:

$$
\left\{\begin{aligned}
\dot{z}(t) & =\sum_{i=1}^{h} \rho_{i}(\theta(t))\left[N_{i} z(t)+G_{i} u_{f}(t)+L_{i} y(t)+B_{i} \hat{f}(t)\right] \\
\hat{x}(t) & =z(t)+T_{2} y(t) \\
\hat{y}(t) & =C \hat{x}(t) \\
\dot{\hat{f}}(t) & =\Gamma \sum_{i=1}^{h} \rho_{i}(\theta(t)) \Phi_{i}\left(\dot{e}_{y}(t)+\sigma e_{y}(t)\right) \\
e_{y}(t) & =y(t)-\hat{y}(t)
\end{aligned}\right.
$$

where $z(t)$ is the observer state vector, $\hat{x}(t)$ the estimated state vector, $\hat{y}(t)$ is the estimated output vector, $\hat{f}(t)$ is the estimated actuator fault and $e_{y}(t)$ is the output estimation error. $N_{i} \in \Re^{n \times n}, G_{i} \in \Re^{n \times p}, L_{i} \in \Re^{n \times m}, \Phi_{i} \in \Re^{p \times m}$ and $T_{2} \in \Re^{n \times m}$ are unknown matrices to be determined afterwards. The matrix $\Gamma \in \Re^{p \times p}$ is a symmetric positive definite learning rate matrix. And $\sigma$ is a positive scalar.

In the remainder of this note, the only necessary assumptions are the following: 
Assumption 1

$$
\operatorname{rank}\left(C B_{i}\right)=\operatorname{rank}\left(B_{i}\right)=p ; \forall i=[1, \ldots, h]
$$

Assumption 2 The polytopic LPV system (5) is observable, ie:

$$
\operatorname{rank}\left(\begin{array}{c}
C \\
C A_{i} \\
\vdots \\
C A_{i}^{n-1}
\end{array}\right)=n ; \forall i=1, \cdots, h
$$

Assumption 3 The polytopic LPV system (5) is detectable, ie:

$$
\operatorname{rank}\left(\begin{array}{c}
s I_{n}-A_{i} \\
C
\end{array}\right)=n ; \forall i=1, \cdots, h \text { where } s \in C
$$

Assumption 4 The input vector $u(t)$ is bounded as $\|\mathrm{u}(t)\| \leq \alpha_{1}$. The fault $f(t)$ satisfies $\|\mathrm{f}(t)\| \leq \alpha_{2}$, the norm of its derivative is bounded i.e. $\|\dot{\mathrm{f}}(t)\| \leq \alpha_{3}$ and the vector $d(t)$ satisfies also $\|d(t)\| \leq \alpha_{4}$, where $\alpha_{1}, \alpha_{2}, \alpha_{3}, \alpha_{4} \geq 0$.

In the following, the aim is to design an Active Fault Tolerant Controller ensuring the tracking trajectory performance of the faulty LPV system to the reference model. The structure of this FTC law is given by:

$$
u_{f}(t)=\sum_{i=1}^{h} \rho_{i}(\theta(t))\left(K_{P i} e_{y r}(t)+K_{I i} \int_{0}^{\tau} e_{y r}(t) d t-H_{i} \hat{f}(t)\right)
$$

where $K_{P i} \in \Re^{m \times n}, K_{I i} \in \Re^{m \times n}$ and $H_{i} \in \Re^{m \times p}$ are control gain matrices to be designed.

Contrary to (Aouaouda et al.) in [1] where the developed approach is based on the design of a controller whose FTC law is considered as an adaptive state feedback control law, the proposed AFTC law (10) is described by an adaptive Proportional Integral (PI) control. This control law improves both the stability and accuracy performances and compensates the fault effects on the studied system.

\section{Tracking Fault Tolerant Control}

\subsection{Reconfiguration analysis}

Let define the reconfigured model as an augmented model which includes principally the state vector $x(t)$, the state of the tracking error $e_{t}(t)$, the state of the estimation error $e_{s}(t)$ and the fault estimation error $e_{f}(t)$. These errors are described by:

$$
\begin{aligned}
& e_{t}(t)=x_{r}(t)-x(t) \\
& e_{s}(t)=x(t)-\hat{x}(t) \\
& e_{f}(t)=f(t)-\hat{f}(t)
\end{aligned}
$$

According to [4], we consider a new state vector $x_{t}(t)$ which is defined by:

$$
x_{t}(t)=\int_{0}^{t} e_{t}(\tau) d \tau
$$


such that its dynamic is expressed as follows:

$$
\dot{x_{t}}(t)=e_{t}(t)
$$

By using the state space representations (3) and (5) and the equation (11), the expression of $e_{y r}(t)$ becomes:

$$
\begin{aligned}
e_{y r}(t) & =y_{r}(t)-y(t) \\
& =C e_{t}(t)
\end{aligned}
$$

Now, tacking into account the equations (13), (14) and (16), the AFTC law (10) can be rewritten as:

$$
u_{f}(t)=\sum_{i=1}^{h} \rho_{i}(\theta(t))\left(K_{P i} C e_{t}(t)+K_{I i} C x_{t}(t)+H_{i} e_{f}(t)-H_{i} f(t)\right)
$$

Substituting (17) into (3), it may be possible to rewrite the dynamic of the state vector $x(t)$ as follows:

$$
\begin{aligned}
\dot{x}(t)= & \sum_{i, j=1}^{h} \rho_{i}(\theta(t)) \rho_{j}(\theta(t))\left[A_{i} x(t)+B_{i} K_{P j} C e_{t}(t)+B_{i} K_{I j} x_{t}(t)+B_{i} H_{j} e_{f}(t)\right. \\
& \left.-H_{i j} f(t)+R_{i} d(t)\right]
\end{aligned}
$$

where,

$$
H_{i j}=B_{i} H_{j}-B_{i}
$$

The state dynamic of the tracking error is:

$$
\dot{e}_{t}(t)=\dot{x}_{r}(t)-\dot{x}(t)
$$

Substituting the expressions of $\dot{x}_{r}(t)$ and $\dot{x}(t)$ respectively from (5) and (18) into (20), which becomes:

$$
\begin{aligned}
\dot{e}_{t}(t)= & \sum_{i, j=1}^{h} \rho_{i}(\theta(t)) \rho_{j}(\theta(t))\left[\left(A_{i}-B_{i} K_{P j} C\right) e_{t}(t)-B_{i} K_{I j} C x_{t}(t)-B_{i} H_{j} e_{f}(t)\right. \\
& \left.+B_{i} u(t)+H_{i j} f(t)-R_{i} d(t)\right]
\end{aligned}
$$

In order to study the state estimation error $e_{s}(t)$ defined by (12), we assume that there exist $T_{1} \in \Re^{n \times n}$ and $T_{2} \in \Re^{n \times m}$ which verify the following equation:

$$
T_{1}+T_{2} C=I_{n}
$$

Therefore the above equation can be rewritten as:

$$
\left[\begin{array}{ll}
T_{1} & T_{2}
\end{array}\right]\left[\begin{array}{l}
I_{n} \\
C
\end{array}\right]=I_{n}
$$

A particular solution of the matrices $T_{1}$ and $T_{2}$ is computed as:

$$
\left[\begin{array}{ll}
T_{1} & T_{2}
\end{array}\right]=\left[\begin{array}{l}
I_{n} \\
C
\end{array}\right]^{+}
$$

Thus, by using (6) and (22), the equation (12) will be rewritten as:

$$
e_{s}(t)=T_{1} x(t)-z(t)
$$


The the state dynamic of the estimation error (25) is then described as follows:

$$
\dot{e}_{s}(t)=T_{1} \dot{x}(t)-\dot{z}(t)
$$

By using the expressions of $\dot{x}(t)$ and $\dot{z}(t)$ respectively from (3) and (6), the last equation (26) can be rewritten as:

$$
\begin{aligned}
\dot{e}_{s}(t)= & \sum_{i=1}^{h} \rho_{i}(\theta(t))\left[\left(T_{1} A_{i}+E_{i} C-N_{i}\right) x(t)+\left(T_{1} B_{i}-G_{i}\right) u(t)+N_{i} e_{s}(t)+B_{i} e_{f}(t)\right. \\
& \left.+M_{i} f(t)+\bar{R}_{i} d(t)\right]
\end{aligned}
$$

where,

$$
\begin{array}{ll} 
& E_{i}=N_{i} T_{2}-L_{i} \\
& M_{i}=T_{1} B_{i}-B_{i} \\
\text { and } \quad & \bar{R}_{i}=T_{1} R_{i}
\end{array}
$$

We assume that for all $i=1, \ldots, h$, the following conditions hold true:

$$
\begin{array}{ll} 
& T_{1} A_{i}+E_{i} C-N_{i}=0 \\
\text { and } & T_{1} B_{i}-G_{i}=0
\end{array}
$$

Therefore, by taking into account (31) and (32), the state dynamic of the estimation error (27) holds:

$$
\dot{e}_{s}(t)=\sum_{i=1}^{h} \rho_{i}(\theta(t))\left[N_{i} e_{s}(t)+B_{i} e_{f}(t)+M_{i} f(t)+\bar{R}_{i} d(t)\right]
$$

The fault estimation error dynamic can be expressed as follows:

$$
\dot{e}_{f}(t)=\dot{f}(t)-\dot{\hat{f}}(t)
$$

\subsection{Stability analysis}

Before beginning the stability analysis, we consider the following lemma:

Lemma 1 Given a symmetric positive matrix $P$ and a positive scalar $\mu$, the following inequality holds [10]:

$$
2 x^{T} y \leq \frac{1}{\mu} x^{T} P x+\mu y^{T} P^{-1} y ; x, y \in \Re^{n}
$$

Lemma 2 Let $X, Y$ and $Z$ be real matrices of appropriate dimensions with $\Delta$ satisfying $\Delta^{T} \Delta \leq$ $I$, then [7]:

$$
X+Y \Delta Z+Z^{T} \Delta^{T} Y^{T} \prec 0
$$

if and only if there exists a positive scalar $\psi \succ 0$ such that:

$$
X+\psi Z^{T} Z+\frac{1}{\psi} Y Y^{T} \prec 0
$$

or equivalently:

$$
\left[\begin{array}{ccc}
X & Y & \psi Z^{T} \\
Y^{T} & -\psi I & 0 \\
\psi Z & 0 & -\psi I
\end{array}\right] \prec 0
$$


For effective Active Fault Tolerant Control, the reconfigured model presented in the last section must be stable in order to prove that the states of the faulty LPV system converge asymptotically to the states of the reference model. The following theorem provides sufficient conditions to fulfill this goal.

Theorem 1 Let consider the polytopic system (3), the Active Fault Tolerant Control (AFTC) (10), the reference model (5) and the Adaptive Polytopic Observer (AOP) (6). Given positive scalars $\sigma, \mu, \beta$ and $\psi$ and a symmetric and positive definite matrix $\Gamma$, the faulty state $x(t)$, the state of the tracking error $e_{t}(t)$, the state of the estimation error $e_{s}(t)$ and the fault estimation error $e_{f}(t)$ are bounded if there exist symmetric and positive definite matrices $X_{1}=P_{1}^{-1}$, $X_{2}=P_{2}^{-1}, X_{3}=P_{3}^{-1}, Q_{1}, Q_{2}, Q_{3}$ and $Q_{4}$ and matrices $W_{P j}=K_{P j} C X_{2}, W_{I j}=K_{I j} C X_{2}$, $S_{i}=E_{i} C X_{3}, H_{i}$ and $\Phi_{i}$ such that the following LMIs are satisfied for all $i, j, k=1, \ldots, h$ :

$$
\left(\begin{array}{cccc}
\bar{X}_{i j k} & Y_{i} & \psi Z_{k}^{T} & P \\
* & -\psi I & 0 & 0 \\
* & * & -\psi I & 0 \\
* & * & * & -Q
\end{array}\right) \prec 0
$$

where,

$$
\begin{aligned}
& \bar{X}_{i j k}=\left(\begin{array}{ccccc}
\Pi_{i} & B_{i} W_{P j} & B_{i} W_{I j} & 0 & B_{i} H_{j} \\
* & \Omega_{i j} & X_{2}-B_{i} W_{I j} & 0 & -B_{i} H_{j} \\
* & * & -\beta I & 0 & 0 \\
* & * & * & \Theta_{i} & B_{i} \\
* & * & * & * & \Sigma_{i k}
\end{array}\right) \\
& Y_{i}=\left(\begin{array}{ccccc}
0 & 0 & 0 & 0 & 0 \\
0 & 0 & 0 & 0 & 0 \\
0 & 0 & 0 & 0 & 0 \\
0 & 0 & 0 & 0 & 0 \\
0 & 0 & -\Phi_{i} C & 0 & 0
\end{array}\right) \\
& Z_{k}^{T}=\left(\begin{array}{ccccc}
0 & 0 & 0 & 0 & 0 \\
0 & 0 & 0 & 0 & 0 \\
0 & 0 & 0 & 0 & 0 \\
0 & 0 & \Xi_{k}^{T} & 0 & 0 \\
0 & 0 & 0 & 0 & 0
\end{array}\right) \\
& P=\left(\begin{array}{cccc}
X_{1}{ }^{T} & 0 & 0 & 0 \\
0 & X_{2}{ }^{T} & 0 & 0 \\
0 & 0 & I & 0 \\
0 & 0 & 0 & X_{3}^{T} \\
0 & 0 & 0 & 0
\end{array}\right)
\end{aligned}
$$

and

$$
Q=\left(\begin{array}{cccc}
\frac{2}{\mu} Q_{1} & 0 & 0 & 0 \\
* & \frac{3}{\mu} Q_{2} & 0 & 0 \\
* & * & \beta I & 0 \\
* & * & * & \frac{2}{\mu} Q_{3}
\end{array}\right)
$$


with,

$$
\begin{aligned}
& \Pi_{i}=A_{i} X_{1}+X_{1} A_{i}^{T} \\
& \Omega_{i j}=A_{i} X_{2}+X_{2} A_{i}^{T}-B_{i} W_{P j}-W_{P j}^{T} B_{i}^{T} \\
& \Theta_{i}=T_{1} A_{i} X_{3}+X_{3} A_{i}^{T} T_{1}^{T}+S_{i}+S_{i}^{T} \\
& \Xi_{k}=X_{3}+\frac{1}{\sigma}\left(T_{1} A_{k} X_{3}+S_{k}\right) \\
& \Sigma_{i k}=-\frac{1}{\sigma}\left(\Phi_{i} C B_{k}+B_{k}^{T} C^{T} \Phi_{i}^{T}\right)+\frac{3}{\mu \sigma} Q_{4}
\end{aligned}
$$

The controller and the observer gains are given by:

$$
\begin{array}{ll} 
& K_{P j}=W_{P j}\left(C X_{2}\right)^{-1} \\
& K_{I j}=W_{I j}\left(C X_{2}\right)^{-1} \\
& N_{i}=T_{1} A_{i}+E_{i} C \\
& L_{i}=N_{i} T_{2}-E_{i} \\
\text { and } \quad & G_{i}=T_{1} B_{i}
\end{array}
$$

Proof 1 In this proof, we use the Lyapunov's method to prove the stability of the reconfigured model whose states are $x(t), e_{t}(t), x_{t}(t) e_{s}(t)$ and $e_{f}(t)$. Moreover, we will formulate the LMIs in order to solve the optimisation problem and determinate the unknown matrices of both the controller and the observer.

Consider a quadratic Lyapunov function defined as follows:

$$
V(t)=x^{T}(t) P_{1} x(t)+e_{t}^{T}(t) P_{2} e_{t}(t)+x_{t}^{T}(t) P_{2} x_{t}(t)+e_{s}^{T}(t) P_{3} e_{s}(t)+\frac{1}{\sigma} e_{f}^{T}(t) \Gamma^{-1} e_{f}(t)
$$

where $P_{1}, P_{2}, P_{3}$ and $\Gamma^{-1}$ are symmetic positive definite matrices with appropriate dimensions. The time derivative of the above Lyapunov function (55) yields:

$$
\begin{aligned}
\dot{V}(t) & =\dot{x}^{T}(t) P_{1} x(t)+x^{T}(t) P_{1} \dot{x}(t)+\dot{e}_{t}^{T}(t) P_{2} e_{t}(t)+e_{t}^{T}(t) P_{2} \dot{e}_{t}(t)+\dot{x}_{t}^{T}(t) P_{2} x_{t}(t) \\
& +x_{t}^{T}(t) P_{2} \dot{x}_{t}(t)+\dot{e}_{s}^{T}(t) P_{3} e_{s}(t)+e_{s}^{T}(t) P_{3} \dot{e}_{s}(t)+\frac{1}{\sigma} \dot{e}_{f}^{T}(t) \Gamma^{-1} e_{f}(t)+\frac{1}{\sigma} e_{f}^{T}(t) \Gamma^{-1} \dot{e}_{f}(t)
\end{aligned}
$$

By taking into account the expressions (18) of $\dot{x}(t)$, (21) of $\dot{e}_{t}(t)$, (15) of $\dot{x}_{t}(t)$, (33) of $\dot{e}_{s}(t)$ and (34) of $\dot{e}_{f}(t)$ and using the expression of $\dot{\hat{f}}(t)$ in (6), the equation (56) becomes:

$$
\begin{aligned}
\dot{V}(t) & =\sum_{i, j=1}^{h} \rho_{i}(\theta(t)) \rho_{j}(\theta(t))\left[x^{T}(t)\left(A_{i}^{T} P_{1}+P_{1} A_{i}\right) x(t)+2 x^{T}(t) P_{1} B_{i} K_{P j} C e_{t}(t)\right. \\
& +2 x^{T}(t) P_{1} B_{i} K_{I j} C x(t)+2 x^{T}(t) P_{1} B_{i} H_{j} e_{f}(t)-2 x^{T}(t) P_{1} H_{i j} f(t)+2 x^{T}(t) P_{1} R_{i} d(t) \\
& +e_{t}^{T}(t)\left(A_{i j}{ }^{T} P_{2}+P_{2} A_{i j}\right) e_{t}(t)+2 e_{t}^{T}(t)\left(P_{2}-P_{2} B_{i} K_{I j} C\right) x_{t}(t)-2 e_{t}^{T}(t) P_{2} B_{i} H_{j} e_{f}(t) \\
& +2 e_{t}^{T}(t) P_{2} H_{i j} f(t)+2 e_{t}^{T}(t) P_{2} B_{i} u(t)-2 e_{t}^{T}(t) P_{2} R_{i} d(t)+e_{s}^{T}(t)\left(N_{i}^{T} P_{3}+P_{3} N_{i}\right) e_{s}(t) \\
& +2 e_{s}^{T}(t)\left(P_{3} B_{i}-C^{T} \Phi_{i}^{T}\right) e_{f}(t)+2 e_{s}^{T}(t) P_{3} M_{i} f(t)+2 e_{s}^{T}(t) P_{3} \bar{R}_{i} d(t) \\
& \left.-\frac{1}{\sigma} \dot{e}_{s}^{T}(t) C^{T} \Phi_{i}^{T} e_{f}(t)-\frac{1}{\sigma} e_{f}^{T}(t) \Phi_{i} C \dot{e}_{s}(t)+\frac{2}{\sigma} e_{f}^{T}(t) \Gamma^{-1} \dot{f}(t)\right]
\end{aligned}
$$

where,

$$
A_{i j}=A_{i}-B_{i} K_{P j} C
$$


Now, substituting $\dot{e}_{s}(t)$ by its expression (33), the equation (57) will be rewritten as follows:

$$
\begin{aligned}
\dot{V}(t) & =\sum_{i, j, k=1}^{h} \rho_{i}(\theta(t)) \rho_{j}(\theta(t)) \rho_{k}(\theta(t))\left[x^{T}(t)\left(A_{i}^{T} P_{1}+P_{1} A_{i}\right) x(t)+2 x^{T}(t) P_{1} B_{i} K_{P j} C e_{t}(t)\right. \\
& +2 x^{T}(t) P_{1} B_{i} K_{I j} C x_{t}(t)+2 x^{T}(t) P_{1} B_{i} H_{j} e_{f}(t)-2 x^{T}(t) P_{1} H_{i j} f(t)+2 x^{T}(t) P_{1} R_{i} d(t) \\
& +e_{t}^{T}(t)\left(A_{i j}{ }^{T} P_{2}+P_{2} A_{i j}\right) e_{t}(t)+2 e_{t}^{T}(t)\left(P_{2}-P_{2} B_{i} K_{I j} C\right) x_{t}(t)-2 e_{t}^{T}(t) P_{2} B_{i} H_{j} e_{f}(t) \\
& +2 e_{t}^{T}(t) P_{2} H_{i j} f(t)+2 e_{t}^{T}(t) P_{2} B_{i} u(t)-2 e_{t}^{T}(t) P_{2} R_{i} d(t)+e_{s}^{T}(t)\left(N_{i}^{T} P_{3}+P_{3} N_{i}\right) e_{s}(t) \\
& +2 e_{s}^{T}(t)\left(P_{3} B_{i}-C^{T} \Phi_{i}^{T}-\frac{1}{\sigma} N_{k}^{T} C^{T} \Phi_{i}^{T}\right) e_{f}(t)+2 e_{s}^{T}(t) P_{3} M_{i} f(t)+2 e_{s}{ }^{T}(t) P_{3} \bar{R}_{i} d(t) \\
& -\frac{1}{\sigma} e_{f}^{T}(t)\left(\Phi_{i} C B_{k}+B_{k}{ }^{T} C^{T} \Phi_{i}{ }^{T}\right) e_{f}(t)-\frac{2}{\sigma} e_{f}^{T}(t) \Phi_{i} C M_{k} f(t)+\frac{2}{\sigma} e_{f}^{T}(t) \Phi_{i} C \bar{R}_{k} d(t) \\
& \left.+\frac{2}{\sigma} e_{f}^{T}(t) \Gamma^{-1} \dot{f}(t)\right]
\end{aligned}
$$

By applying the Lemma 1 and using the Assumption 4, the following term from the equation (59) can be bounded as follows:

$$
\begin{aligned}
-2 x^{T}(t) P_{1} H_{i j} f(t) & \leq \frac{1}{\mu} x^{T}(t) Q_{1} x(t)+\mu f^{T}(t) H_{i j}{ }^{T} P_{1} Q_{1}{ }^{-1} P_{1} H_{i j} f(t) \\
& \leq \frac{1}{\mu} x^{T}(t) Q_{1} x(t)+\eta_{1 i j}
\end{aligned}
$$

In the same manner, the other terms may be bounded as:

$$
\begin{aligned}
& 2 x^{T}(t) P_{1} R_{i} d(t) \leq \frac{1}{\mu} x^{T}(t) Q_{1} x(t)+\eta_{2 i} \\
& 2 e_{t}^{T}(t) P_{2} B_{i} u(t) \leq \frac{1}{\mu} e_{t}^{T}(t) Q_{2} e_{t}(t)+\eta_{3 i} \\
& 2 e_{t}^{T}(t) P_{2} H_{i j} f(t) \leq \frac{1}{\mu} e_{t}^{T}(t) Q_{2} e_{t}(t)+\eta_{4 i j} \\
& -2 e_{t}^{T}(t) P_{2} R_{i} d(t) \leq \frac{1}{\mu} e_{t}^{T}(t) Q_{2} e_{t}(t)+\eta_{5 i} \\
& 2 e_{s}^{T}(t) P_{3} M_{i} f(t) \leq \frac{1}{\mu} e_{s}^{T}(t) Q_{3} e_{s}(t)+\eta_{6 i} \\
& 2 e_{s}^{T}(t) P_{3} \bar{R}_{i} d(t) \leq \frac{1}{\mu} e_{s}^{T}(t) Q_{3} e_{s}(t)+\eta_{7 i} \\
& -\frac{2}{\sigma} e_{f}^{T}(t) \Phi_{i} C M_{k} f(t) \leq \frac{1}{\mu} e_{f}^{T}(t) Q_{4} e_{f}(t)+\eta_{8 i k} \\
& \frac{2}{\sigma} e_{f}^{T}(t) \Phi_{i} C \bar{R}_{k} d(t) \leq \frac{1}{\mu} e_{f}^{T}(t) Q_{4} e_{f}(t)+\eta_{9 i k} \\
& \frac{2}{\sigma} e_{f}^{T}(t) \Gamma^{-1} \dot{f}(t) \leq \frac{1}{\mu} e_{f}^{T}(t) Q_{4} e_{f}(t)+\eta_{10}
\end{aligned}
$$

with, 


$$
\begin{gathered}
\eta_{1 i j}=\mu \alpha_{2}{ }^{2} \lambda_{\max }\left(H_{i j}^{T} P_{1} Q_{1}{ }^{-1} P_{1} H_{i j}\right) \\
\eta_{2 i}=\mu \alpha_{4}{ }^{2} \lambda_{\max }\left(R_{i}^{T} P_{1} Q_{1}{ }^{-1} P_{1} R_{i}\right) \\
\eta_{3 i}=\mu \alpha_{1}{ }^{2} \lambda_{\max }\left(B_{i}^{T} P_{2} Q_{2}{ }^{-1} P_{2} B_{i}\right) \\
\eta_{4 i j}=\mu \alpha_{2}{ }^{2} \lambda_{\max }\left(F_{i j}{ }^{T} P_{2} Q_{2}{ }^{-1} P_{2} F_{i j}\right) \\
\eta_{5 i}=\mu \alpha_{4}{ }^{2} \lambda_{\max }\left(R_{i}^{T} P_{2} Q_{2}{ }^{-1} P_{2} R_{i}\right) \\
\eta_{6 i}=\mu \alpha_{2}{ }^{2} \lambda_{\max }\left(M_{i}^{T} P_{3} Q_{3}{ }^{-1} P_{3} M_{i}\right) \\
\eta_{7 i}=\mu \alpha_{4}{ }^{2} \lambda_{\max }\left(\bar{R}_{i}^{T} P_{3} Q_{3}{ }^{-1} P_{3} \bar{R}_{i}\right) \\
\eta_{8 i k}=\mu \alpha_{2}{ }^{2} \lambda_{\max }\left(M_{k}{ }^{T} C^{T} \Phi_{i}{ }^{T} Q_{4}{ }^{-1} \Phi_{i} C M_{k}\right) \\
\eta_{9 i k}=\mu \alpha_{4}{ }^{2} \lambda_{\max }\left(\bar{R}_{k}^{T} C^{T} \Phi_{i}{ }^{T} Q_{4}{ }^{-1} \Phi_{i} C \bar{R}_{k}\right) \\
\eta_{10}=\mu \alpha_{3}{ }^{2} \lambda_{\max }\left(\Gamma^{-1^{T}} Q_{4}{ }^{-1} \Gamma^{-1}\right)
\end{gathered}
$$

By taking into account the inequalities (60)-(69), $\dot{V}(t)$ can be bounded as follows:

$$
\begin{aligned}
\dot{V}(t) & \leq \sum_{i, j, k=1}^{h} \rho_{i}(\theta(t)) \rho_{j}(\theta(t)) \rho_{k}(\theta(t))\left[x^{T}(t)\left(A_{i}^{T} P_{1}+P_{1} A_{i}+\frac{2}{\mu} Q_{1}\right) x(t)\right. \\
& +2 x^{T}(t) P_{1} B_{i} K_{P j} C e_{t}(t)+2 x^{T}(t) P_{1} B_{i} K_{I j} C x_{t}(t)+2 x^{T}(t) P_{1} B_{i} H_{j} e_{f}(t) \\
& +e_{t}^{T}(t)\left(A_{i j}^{T} P_{2}+P_{2} A_{i j}+\frac{3}{\mu} Q_{2}\right) e_{t}(t)+2 e_{t}^{T}(t)\left(P_{2}-P_{2} B_{i} K_{I j} C\right) x_{t}(t) \\
& -2 e_{t}^{T}(t) P_{2} B_{i} H_{j} e_{f}(t)+e_{s}^{T}(t)\left(N_{i}^{T} P_{3}+P_{3} N_{i}+\frac{2}{\mu} Q_{3}\right) e_{s}(t)+2 e_{s}^{T}(t)\left(P_{3} B_{i}-C^{T} \Phi_{i}^{T}\right. \\
& \left.\left.-\frac{1}{\sigma} N_{k}^{T} C^{T} \Phi_{i}^{T}\right) e_{f}(t)-\frac{1}{\sigma} e_{f}^{T}(t)\left(\Phi_{i} C B_{k}+B_{k}^{T} C^{T} \Phi_{i}^{T}+\frac{3}{\mu \sigma} Q_{4}\right) e_{f}(t)\right]+\delta
\end{aligned}
$$

where the scalar $\delta$ is the maximum value over $\mathrm{i}, \mathrm{j}$ and $\mathrm{k}$ such that:

$$
\delta=\max _{i, j, k}\left(\eta_{1 i j}+\eta_{2 i}+\eta_{3 i}+\eta_{4 i j}+\eta_{5 i}+\eta_{6 i}+\eta_{7 i}+\eta_{8 i k}+\eta_{9 i k}+\eta_{10}\right)
$$

Then, the inequality (80) can be rewritten under the following form:

$$
\dot{V}(t) \leq \tilde{x}^{T}(t)\left(\sum_{i, j, k=1}^{h} \rho_{i}(\theta(t)) \rho_{j}(\theta(t)) \rho_{k}(\theta(t)) \Lambda_{i j k}\right) \tilde{x}(t)+\delta
$$

where $\tilde{x}(t)=\left[\begin{array}{lllll}x^{T}(t) & e_{t}^{T}(t) & x_{t}^{T}(t) & e_{s}^{T}(t) & e_{f}^{T}(t)\end{array}\right]^{T}$ is an augmented system and $\Lambda_{i j k}$ is a matrix defined as follows:

$$
\Lambda_{i j k}=\left(\begin{array}{ccccc}
\tilde{\Pi}_{i} & P_{1} B_{i} K_{P j} C & P_{1} B_{i} K_{I j} C & 0 & P_{1} B_{i} H_{j} \\
* & \tilde{\Omega}_{i j} & P_{2}-P_{2} B_{i} K_{I j} C & 0 & -P_{2} B_{i} H_{j} \\
* & * & 0 & 0 & 0 \\
* & * & * & \tilde{\Theta}_{i} & \tilde{\Xi}_{i k} \\
* & * & * & * & \Sigma_{i k}
\end{array}\right)
$$


with,

$$
\begin{aligned}
\tilde{\Pi}_{i} & =A_{i}{ }^{T} P_{1}+P_{1} A_{i}+\frac{2}{\mu} Q_{1} \\
\tilde{\Omega}_{i j} & =A_{i j}{ }^{T} P_{2}+P_{2} A_{i j}+\frac{3}{\mu} Q_{2} \\
\tilde{\Theta}_{i} & =N_{i}{ }^{T} P_{3}+P_{3} N_{i}+\frac{2}{\mu} Q_{3} \\
\tilde{\Xi}_{i k} & =P_{3} B_{i}-C^{T} \Phi_{i}{ }^{T}-\frac{1}{\sigma} N_{k}{ }^{T} C^{T} \Phi_{i}{ }^{T} \\
\text { and } \quad \Sigma_{i k} & =-\frac{1}{\sigma}\left(\Phi_{i} C B_{k}+B_{k}{ }^{T} C^{T} \Phi_{i}{ }^{T}\right)+\frac{3}{\mu \sigma} Q_{4}
\end{aligned}
$$

If the following constraint holds:

$$
\sum_{i, j, k=1}^{h} \rho_{i}(\theta(t)) \rho_{j}(\theta(t)) \rho_{k}(\theta(t)) \Lambda_{i j k} \prec 0
$$

we can obtain:

$$
\dot{V}(t) \leq-\varepsilon\|\tilde{x}(t)\|^{2}+\delta
$$

where $\varepsilon$ is a positive scalar defined by:

$$
\varepsilon=\min \lambda_{\min }\left(-\sum_{i, j, k=1}^{h} \rho_{i}(\theta(t)) \rho_{j}(\theta(t)) \rho_{k}(\theta(t)) \Lambda_{i j k}\right)
$$

and it can be bounded as follows:

$$
\varepsilon \leq \min _{i, j, k} \lambda_{\min }\left(-\Lambda_{i j k}\right)
$$

Consequently, we can say that $\dot{V}(t) \prec 0$, if $\varepsilon\|\tilde{x}(t)\|^{2} \succ \delta ; \forall t \geq 0$. Based on the Lyapunov stability theory, this proves that the augmented system $\tilde{x}(t)$ is stable meaning that the faulty state vector $x(t)$, the state vector $x_{t}(t)$, the state of the tracking error $e_{t}(t)$, the state of the estimation error $e_{s}(t)$ and the fault estimation error $e_{f}(t)$ are bounded.

By considering the constraint (89), we define a matrix:

$$
X=\left(\begin{array}{ccccc}
P_{1}^{-1} & 0 & 0 & 0 & 0 \\
* & P_{2}{ }^{-1} & 0 & 0 & 0 \\
* & * & P_{2}{ }^{-1} & 0 & 0 \\
* & * & * & P_{3}^{-1} & 0 \\
* & * & * & * & I
\end{array}\right) \succ 0
$$

such that $\Psi_{i j k}=X \Lambda_{i j k} X \prec 0$.

Then, considering the following change of variables:

$$
\begin{aligned}
& X_{1}=P_{1}^{-1} \\
& X_{2}=P_{2}^{-1} \\
& X_{3}=P_{3}{ }^{-1}
\end{aligned}
$$


and computing $\Psi_{i j k} \prec 0$ that holds:

$$
\Psi_{i j k}=\left(\begin{array}{ccccc}
X_{1} \tilde{\Pi}_{i} X_{1} & B_{i} K_{P j} C X_{2} & B_{i} K_{I j} C X_{2} & 0 & B_{i} H_{j} \\
* & X_{2} \tilde{\Omega}_{i j} X_{2} & X_{2}-B_{i} K_{I j} C X_{2} & 0 & -B_{i} H_{j} \\
* & * & 0 & 0 & 0 \\
* & * & * & X_{3} \tilde{\Theta}_{i} X_{3} & X_{3} \tilde{\Xi}_{i k} \\
* & * & * & * & \Sigma_{i k}
\end{array}\right) \prec 0
$$

where,

$$
\begin{aligned}
& X_{1} \tilde{\Pi}_{i} X_{1}=\Pi_{i}+\frac{2}{\mu} X_{1} Q_{1} X_{1} \\
& X_{2} \tilde{\Omega}_{i j} X_{2}=\Omega_{i j}+\frac{3}{\mu} X_{2} Q_{2} X_{2} \\
& X_{3} \tilde{\Theta}_{i} X_{3}=\Theta_{i}+\frac{2}{\mu} X_{3} Q_{3} X_{3} \\
& X_{3} \tilde{\Xi}_{i k}=B_{i}-X_{3} C^{T} \Phi_{i}{ }^{T}-\frac{1}{\sigma} X_{3} N_{k}{ }^{T} C^{T} \Phi_{i}{ }^{T}
\end{aligned}
$$

with,

$$
\begin{array}{ll} 
& \Pi_{i}=X_{1} A_{i}^{T}+A_{i} X_{1} \\
& \Omega_{i j}=X_{2} A_{i j}{ }^{T}+A_{i j} X_{2} \\
\text { and } \quad & \Theta_{i}=X_{3} N_{i}^{T}+N_{i} X_{3}
\end{array}
$$

For all positive scalar $\beta>0$, replacing the zero in the diagonal of the matrix (97) by the term $(\beta I-\beta I=0)$ and using the expression (101) of $\left(X_{3} \tilde{\Xi}_{i k}\right)$ so as to rewrite (97) as this way:

$$
\Psi_{i j k}=X_{i j k}+Y_{i} I Z_{k}+Z_{k}^{T} I Y_{i}^{T} \prec 0
$$

where,

$$
\begin{gathered}
X_{i j k}=\left(\begin{array}{ccccc}
X_{1} \tilde{\Pi}_{i} X_{1} & B_{i} K_{P j} C X_{2} & B_{i} K_{I j} C & 0 & B_{i} H_{j} \\
* & X_{2} \tilde{\Omega}_{i j} X_{2} & X_{2}-B_{i} K_{I j} C X_{2} & 0 & -B_{i} H_{j} \\
* & * & \beta I-\beta I & 0 & 0 \\
* & * & * & X_{3} \tilde{\Theta}_{i} X_{3} & B_{i} \\
* & * & * & * & \Sigma_{i k}
\end{array}\right) \\
Y_{i}=\left(\begin{array}{ccccc}
0 & 0 & 0 & 0 & 0 \\
0 & 0 & 0 & 0 & 0 \\
0 & 0 & 0 & 0 & 0 \\
0 & 0 & 0 & 0 & 0 \\
0 & 0 & -\Phi_{i} C & 0 & 0
\end{array}\right)
\end{gathered}
$$

and,

$$
Z_{k}=\left(\begin{array}{ccccc}
0 & 0 & 0 & 0 & 0 \\
0 & 0 & 0 & 0 & 0 \\
0 & 0 & 0 & \Xi_{k} & 0 \\
0 & 0 & 0 & 0 & 0 \\
0 & 0 & 0 & 0 & 0
\end{array}\right)
$$

with,

$$
\Xi_{k}=X_{3}+\frac{1}{\sigma} N_{k} X_{3}
$$


Applying the Lemma 2, the inequality (105) holds true if and only if there exits a positive scalar $\psi \succ 0$ such that:

$$
X_{i j k}+\psi Z_{k}^{T} Z_{k}+\frac{1}{\psi} Y_{i} Y_{i}^{T} \prec 0
$$

or equivalently:

$$
\left(\begin{array}{ccc}
X_{i j k} & Y_{i} & \psi Z_{k}^{T} \\
* & -\psi I & 0 \\
* & * & -\psi I
\end{array}\right) \prec 0
$$

Now, we dissociate the term $\frac{2}{\mu} X_{1} Q_{1} X_{1}$ from the inequality (111) which may be reformulated as follows:

$$
\left(\begin{array}{ccc}
\tilde{X}_{i j k} & Y_{i} & \psi Z_{k}^{T} \\
* & -\psi I & 0 \\
* & * & -\psi I
\end{array}\right)-\bar{X}_{1}^{T}\left(-\frac{2}{\mu} Q_{1}\right) \bar{X}_{1} \prec 0
$$

where,

$$
\tilde{X}_{i j k}=\left(\begin{array}{ccccc}
\Pi_{i} & B_{i} K_{P j} C X_{2} & B_{i} K_{I j} C X_{2} & 0 & B_{i} H_{j} \\
* & X_{2} \tilde{\Omega}_{i j} X_{2} & X_{2}-B_{i} K_{I j} C X_{2} & 0 & -B_{i} H_{j} \\
* & * & \beta I-\beta I & 0 & 0 \\
* & * & * & X_{3} \tilde{\Theta}_{i} X_{3} & B_{i} \\
* & * & * & * & \Sigma_{i k}
\end{array}\right)
$$

and,

$$
\begin{aligned}
\bar{X}_{1}= & \left(\begin{array}{ccccccccccc}
X_{1} & 0 & 0 & 0 & 0 & 0 & 0 & 0 & 0 & 0 & \cdots \\
& \cdots & 0 & 0 & 0 & 0 & 0
\end{array}\right) \\
&
\end{aligned}
$$

Applying the modified Schur Lemma in the above inequality (112) and then we repeat the same work succesively for the terms $\frac{3}{\mu} X_{2} Q_{2} X_{2}, I(\beta I) I$ and $\frac{2}{\mu} X_{3} Q_{3} X_{3}$ so as to obtain:

$$
\left(\begin{array}{cccc}
\bar{X}_{i j k} & Y_{i} & \psi Z_{k}^{T} & P \\
* & -\psi I & 0 & 0 \\
* & * & -\psi I & 0 \\
* & * & * & -Q
\end{array}\right) \prec 0
$$

where,

$$
\begin{gathered}
\bar{X}_{i j k}=\left(\begin{array}{ccccc}
\Pi_{i} & B_{i} K_{P j} C X_{2} & B_{i} K_{I j} C X_{2} & 0 & B_{i} H_{j} \\
* & \Omega_{i j} & X_{2}-B_{i} K_{I j} C X_{2} & 0 & -B_{i} H_{j} \\
* & * & -\beta I & 0 & 0 \\
* & * & * & \Theta_{i} & B_{i} \\
* & * & * & * & \Sigma_{i k}
\end{array}\right) \\
P=\left(\begin{array}{cccc}
X_{1}^{T} & 0 & 0 & 0 \\
0 & X_{2}{ }^{T} & 0 & 0 \\
0 & 0 & I & 0 \\
0 & 0 & 0 & X_{3}{ }^{T} \\
0 & 0 & 0 & 0
\end{array}\right)
\end{gathered}
$$

and

$$
Q=\left(\begin{array}{cccc}
\frac{2}{\mu} Q_{1} & 0 & 0 & 0 \\
* & \frac{3}{\mu} Q_{2} & 0 & 0 \\
* & * & \beta I & 0 \\
* & * & * & \frac{2}{\mu} Q_{3}
\end{array}\right)
$$

This completes the proof of the theorem. 
In order to solve numerically the theorem1, the following algorithm is proposed:

Algorithm 1 Algorithm to solve the LMI optimization problem.

- Step 1: Compute the matrices $T_{1}$ and $T_{2}$ from (24).

- Step 2: Choose positive values for the scalars $\sigma, \mu, \beta$ and $\psi$ and a symmetric and positive definite matrix $\Gamma$.

- Step 3: Solve the LMIs (39) by using the LMI Toolbox and obtain the unknown matrices: $X_{1}, X_{2}, X_{3}, Q_{1}, Q_{2}, Q_{3}, Q_{4}, W_{P j}, W_{I j}, H_{j}, S_{i}$ and $\Phi_{i}$.

- Step 4: Compute the matrices $E_{i}$ by: $E_{i}=S_{i}\left(C X_{3}\right)^{-1}$.

- Step 5: Compute the gain matrices $K_{P j}, K_{I j}, N_{i}, L_{i}$ and $G_{i}$ from (50)-(54).

for all $i, j=1, h$.

\section{Application on two-tank process}

\subsection{Two-tank process}

The two-tank process, presented in the figure 2, consists in two liquid tanks that can be filled with two identical, independent pumps that deliver the liquid flows $Q_{1}(t)$ and $Q_{2}(t)$. The tanks are interconnected to each other through a pipe whose flow is $Q_{12}(t)$, while the outflow from the system is located at the second tank and provides a flow $Q_{N 2}(t)$ to the consumer [16]. According

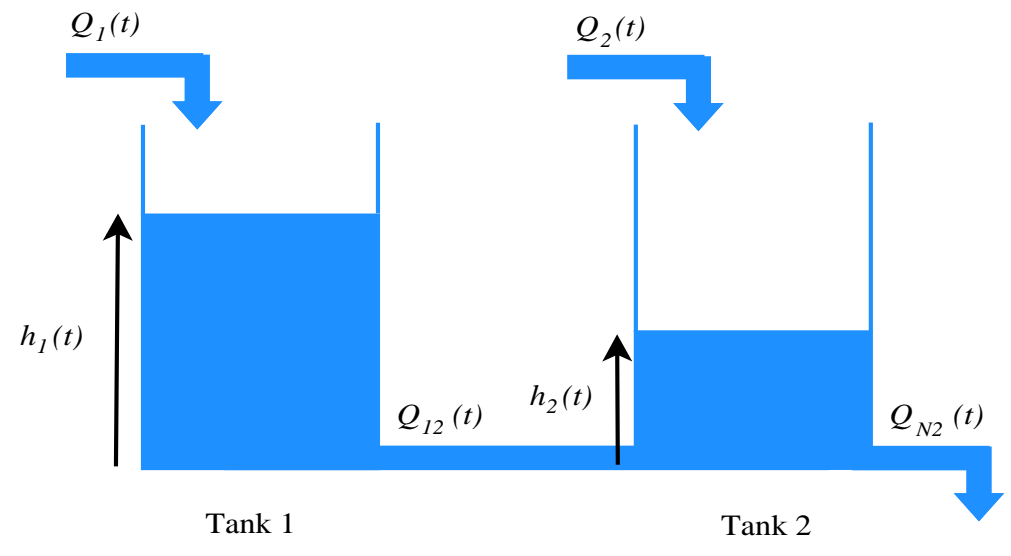

Figure 2: Two-tank process

to [16], using both the mass balance and the Torricellišs law and under the assumption that the system is operating with $h_{1}(t) \succ h_{2}(t)$, the two-tank process can be modeled by the following non-linear state space.

$$
\left\{\begin{aligned}
\frac{d h_{1}(t)}{d t} & =\frac{1}{A}\left(Q_{1}(t)-Q_{12}(t)\right) \\
\frac{d h_{2}(t)}{d t} & =\frac{1}{A}\left(Q_{2}(t)+Q_{12}(t)-Q_{N 2}(t)\right)
\end{aligned}\right.
$$

with,

$$
\begin{gathered}
Q_{12}(t)=c_{12} \sqrt{h_{1}(t)-h_{2}(t)} \\
\text { and, } Q_{N 2}(t)=c_{2} \sqrt{h_{2}(t)}
\end{gathered}
$$


where $h_{1}(t)$ and $h_{2}(t)$ are the liquid levels of the tank 1 and the tank 2 respectively, which are used as state variables. A denotes the area of the cylindric tanks. $c_{12}$ and $c_{2}$ are the constant flows of the interconnecting pipe and of the outflow pipe respectively. The two-tank process's parameters are presented in the table I.

$$
\begin{array}{|cc|c}
\text { Symbol } & \text { Parameters } & \text { Values } \\
A & \text { area of the two tanks } & 1.54 \times 10^{-2} \mathrm{~m}^{2} \\
c_{12} & \text { flow constant of the interconnecting pipe } & 6 \times 10^{-4} \mathrm{~m}^{5 / 2} / \mathrm{s} \\
c_{2} & \text { flow constant of the outflow pipe } & 13 \times 10^{-4} \mathrm{~m}^{5 / 2} / \mathrm{s} \\
\text { process's parameters }
\end{array}
$$

Considering:

$x(t)=\left[\begin{array}{ll}x_{1}(t) & x_{2}(t)\end{array}\right]^{T}=\left[\begin{array}{ll}h_{1}(t) & h_{2}(t)\end{array}\right]^{T}$,

$u(t)=\left[\begin{array}{ll}u_{1}(t) & u_{2}(t)\end{array}\right]^{T}=\left[\begin{array}{ll}Q_{1}(t) & Q_{2}(t)\end{array}\right]^{T}$ and $y(t)=x(t)$ are respectively the state, the control input and the measured output vectors, the two-tank process can be described by the following LPV model:

$$
\left\{\begin{array}{l}
\dot{x}(t)=A(\theta(t)) x(t)+B u(t) \\
y(t)=C x(t)
\end{array}\right.
$$

where the matrices $A(\theta(t)), B$ and $C$ are given by:

$$
\begin{gathered}
A(\theta(t))=\left(\begin{array}{cc}
-a_{11} \theta_{1}(t) & 0 \\
a_{11} \theta_{1}(t) & -a_{22} \theta_{2}(t)
\end{array}\right) ; \\
B=\left(\begin{array}{cc}
b_{11} & 0 \\
0 & b_{22}
\end{array}\right) \text { and } C=\left(\begin{array}{ll}
1 & 0 \\
0 & 1
\end{array}\right)
\end{gathered}
$$

with, $a_{11}=\frac{c_{12}}{A}, a_{22}=\frac{c_{2}}{A}$ and $b_{11}=b_{22}=\frac{1}{A}$

The matrix $A(\theta(t))$ depends on time-varying parameters vector:

$$
\theta(t)=\left(\begin{array}{ll}
\theta_{1}^{T}(t) & \theta_{2}^{T}(t)
\end{array}\right)^{T}
$$

These two time varying parameters $\theta_{1}^{T}(t)$ and $\theta_{2}^{T}(t)$ depend on the measured states $x_{1}(t)$ and $x_{2}(t)$. So, they are said measurable as it is shown in their expressions:

$$
\begin{gathered}
\theta_{1}(t)=\frac{\sqrt{x_{1}(t)-x_{2}(t)}}{x_{1}(t)} \\
\theta_{2}(t)=\frac{\sqrt{x_{2}(t)}}{x_{2}(t)}
\end{gathered}
$$

We consider that the studied process operates with:

$x_{1}(t) \in[0.6 m ; 1.8 m]$ and $x_{2}(t) \in[0.1 m ; 0.3 m]$. In this defined operating range, the time-varying parameters are bounded as follows:

$$
\begin{gathered}
0.6804 m^{-1 / 2} \leq \theta_{1}(t) \leq 1.1785 m^{-1 / 2} \\
\text { and } 1.8257 m^{-1 / 2} \leq \theta_{2}(t) \leq 3.1623 m^{-1 / 2}
\end{gathered}
$$

We consider that additive actuator faults occur in the process's pumps such that the control inputs $u_{1}(t)$ and $u_{2}(t)$ of the faulty pumps are respectively affected by the faults $f_{1}(t)$ and $f_{2}(t)$ which compose the actuator fault vector $f(t)=\left(\begin{array}{lll}f_{1}^{T}(t) & f_{2}{ }^{T}(t)\end{array}\right)^{T}$. Moreover, an unknown input $d(t)$ with a distribution matrix represented by $R=\left[\begin{array}{l}0 \\ 1\end{array}\right]$ is considered in this process. 
Consequently, the whole process can be modeled by the following polytopic LPV model:

$$
\left\{\begin{array}{l}
\dot{x}(t)=\sum_{i=1}^{4} \rho_{i}(\theta(t))\left(A_{i} x(t)+B(u(t)+f(t))+R d(t)\right) \\
y(t)=C x(t)
\end{array}\right.
$$

where the gain scheduling functions are described as follows:

$$
\begin{aligned}
& \rho_{1}(\theta(t))=\frac{\theta_{1}(t)-\underline{\theta_{1}}}{\overline{\theta_{1}}-\underline{\theta_{1}}} \frac{\theta_{2}(t)-\underline{\theta_{2}}}{\overline{\theta_{2}}-\underline{\theta_{2}}} \\
& \rho_{2}(\theta(t))=\frac{\theta_{1}(t)-\underline{\theta_{1}}}{\overline{\theta_{1}}-\underline{\theta_{1}}} \frac{\overline{\theta_{2}}-\theta_{2}(t)}{\overline{\theta_{2}}-\underline{\theta_{2}}} \\
& \rho_{3}(\theta(t))=\frac{\overline{\theta_{1}}-\theta_{1}(t)}{\overline{\theta_{1}}-\underline{\theta_{1}}} \frac{\theta_{2}(t)-\underline{\theta_{2}}}{\overline{\theta_{2}}-\underline{\theta_{2}}} \\
& \rho_{4}(\theta(t))=\frac{\overline{\theta_{1}}-\theta_{1}(t)}{\overline{\theta_{1}}-\underline{\theta_{1}}} \frac{\overline{\theta_{2}}-\theta_{2}(t)}{\overline{\theta_{2}}-\underline{\theta_{2}}}
\end{aligned}
$$

and the matrices $A_{1, . ., 4}$ are given as follows:

$$
\begin{aligned}
& A_{1}=\left(\begin{array}{cc}
-a_{11} \underline{\theta_{1}} & 0 \\
a_{11} \underline{\theta_{1}} & -a_{22} \underline{\theta_{2}}
\end{array}\right) \\
& A_{2}=\left(\begin{array}{cc}
-a_{11} \underline{\theta_{1}} & 0 \\
a_{11} \underline{\theta_{1}} & -a_{22} \overline{\theta_{2}}
\end{array}\right) \\
& A_{3}=\left(\begin{array}{cc}
-a_{11} \overline{\theta_{1}} & 0 \\
a_{11} \overline{\theta_{1}} & -a_{22} \underline{\theta_{2}}
\end{array}\right)
\end{aligned}
$$

and,

$$
A_{4}=\left(\begin{array}{cc}
-a_{11} \overline{\theta_{1}} & 0 \\
a_{11} \overline{\theta_{1}} & -a_{22} \overline{\theta_{2}}
\end{array}\right)
$$

with, $\underline{\theta_{i}}$ and $\overline{\theta_{i}}$ are respectively the minimum and the maximum values of time-varying parameter $\theta_{i}$ for all $i=1,2$.

The above gain scheduling functions $\rho_{1}(\theta(t)), \rho_{2}(\theta(t)), \rho_{3}(\theta(t))$ and $\rho_{4}(\theta(t))$ are plotted in the figure 3.

\subsection{Result simulation}

Solving the LMI (39) by using the LMI Toolbox give the unknown matrices of the controller (10) and the observer (6) through the expressions (50)-(54). For $\mu=1, \sigma=2, \psi=2$ and $\beta=2$, we obtain the matrices $K_{P j}, K_{I j}, H_{j}, N_{i}, L_{i}, G_{i}$ and $\phi_{i}(\forall i, j=1, \ldots, 4)$ which are used in the simulation.

In the simulation, we consider that the flow to the consumer $Q_{N 2}$ is bounded such that:

$$
1.3 \times 10^{-4} \mathrm{~m}^{3} / \mathrm{s} \leq Q_{N 2} \leq 3.9 \times 10^{-4} \mathrm{~m}^{3} / \mathrm{s}
$$




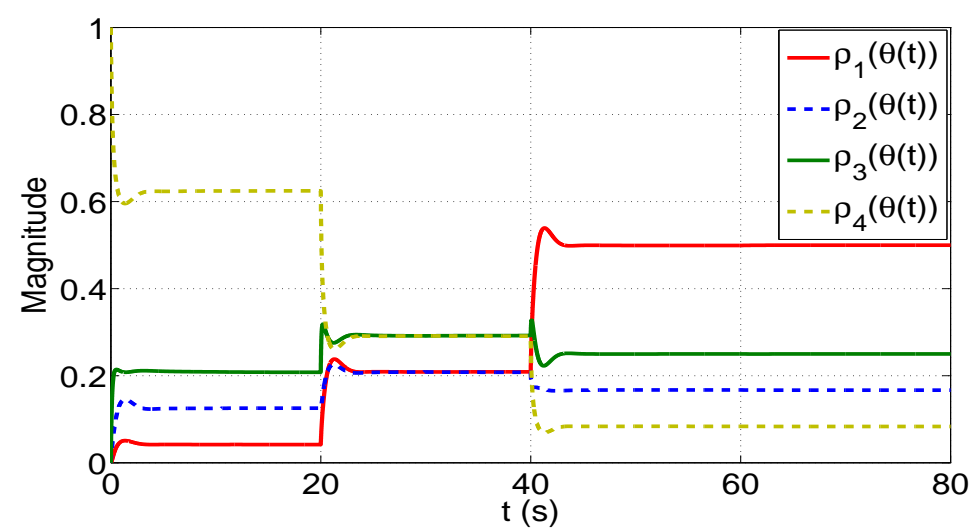

Figure 3: Gain scheduling functions

The references of liquid level in the two tanks are expressed as follows:

$$
h_{r 1}(t)=\left\{\begin{array}{l}
0.8 m \text { for } 0 \mathrm{~s} \leq t \leq 20 s \\
1.1 m \text { for } 20 \mathrm{~s} \prec t \leq 40 s \\
1.4 m \text { for } \mathrm{t} \succ 40 s
\end{array}\right.
$$

and,

$$
h_{r 2}(t)=\left\{\begin{array}{l}
0.15 m \text { for } 0 \mathrm{~s} \leq t \leq 20 s \\
0.20 m \text { for } 20 \mathrm{~s} \prec t \leq 40 s \\
0.25 m \text { for } \mathrm{t} \succ 40 s
\end{array}\right.
$$

The fauts $f_{1}(t)$ and $f_{2}(t)$ affecting the pumps, are considered as incipient faults such that they can be expressed as follows:

$$
f_{1}(t)=\left\{\begin{array}{c}
0 \mathrm{~m}^{3} / \mathrm{s} \text { for } 0 \mathrm{~s} \leq t \prec 10 s \\
-1 \times 10^{-4}(t-10) \mathrm{m}^{3} / \mathrm{s} \text { for } 10 s \leq \mathrm{t} \leq 50 \mathrm{~s} \\
-4 \times 10^{-3} \mathrm{~m}^{3} / \mathrm{s} \text { for } \mathrm{t} \succ 50 \mathrm{~s}
\end{array}\right.
$$

and

$$
f_{2}(t)=\left\{\begin{array}{c}
0 \mathrm{~m}^{3} / \mathrm{s} \text { for } 0 \mathrm{~s} \leq t \prec 30 s \\
-1 \times 10^{-4}(t-10) \mathrm{m}^{3} / \mathrm{s} \text { for } 30 s \leq \mathrm{t} \leq 60 \mathrm{~s} \\
-3 \times 10^{-3} \mathrm{~m}^{3} / \mathrm{s} \text { for } \mathrm{t} \succ 60 \mathrm{~s}
\end{array}\right.
$$

The unknown input $d(t)$ disturbing the second pump through the distribution matrix $\mathrm{R}$, is assumed as a rectangular signal such that:

$$
d(t)=\left\{\begin{array}{c}
1 \times 10^{-3} \mathrm{~m}^{3} / \mathrm{s} \text { for } 15 s \leq t \leq 25 s \\
0 \mathrm{~m}^{3} / \mathrm{s} \text { elsewhere }
\end{array}\right.
$$

The above level references $h_{r 1}(t)$ and $h_{r 2}(t)$, the faults $f_{1}(t)$ and $f_{2}(t)$ and the unknown input $d(t)$ are applied on the closed loop of the two-tank process whose simulation gives the following results. 
The control inputs $u_{f 1}(t)$ and $u_{f 2}(t)$ described by the adaptive PI control law (10) and provided by the proposed active fault tolerant controller for faulty system, are compared to their for healthy system (under no fault) and illustrated in the figure 4.

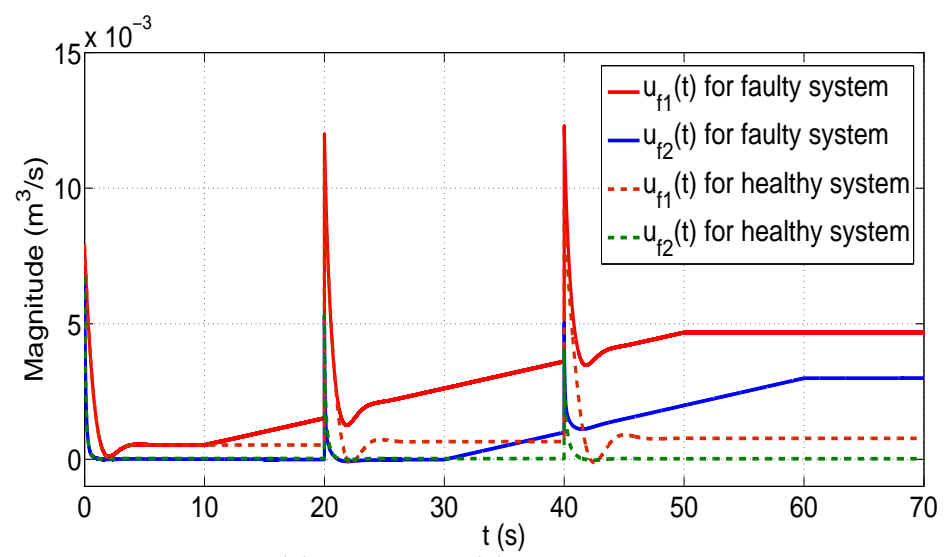

Figure 4: Comparison between the AFTC $u_{f 1}(t)$ and $u_{f 2}(t)$ for faulty system and their for healthy system

Note that the magnitudes of the control inputs $u_{f 1}(t)$ and $u_{f 2}(t)$, for the faulty system, start increasing when the faults occur. And from the time instants $50 \mathrm{~s}$ and $60 \mathrm{~s}$, respectively $u_{f 1}(t)$ and $u_{f 2}(t)$ remain constant. These ones show the fault effects compensation.

In this paper, we compare our results to the FTC approach presented in [1], where the control law is given by:

$$
u_{f}(t)=\sum_{i=1}^{h} \rho_{i}(\theta(t))\left(u(t)+K_{i}\left(x_{r}(t)-x(t)\right)-K_{i}^{f} \hat{f}(t)\right)
$$

The authors in [1] use a PI observer so as to estimate only constant faults and states. So, in order to apply their developed approach on the studied process where the faults are assumed time-varying, we must replace the PI observer by an APO. This allows us to obtain the LMI (39) and the following matrices $\bar{X}_{i j k}, Y_{i}, Z_{k}, P$ and $Q$ :

$$
\begin{gathered}
\bar{X}_{i j k}=\left(\begin{array}{cccc}
\Pi_{i} & B_{i} W_{j} & 0 & B_{i} K_{j}{ }^{f} \\
* & \bar{\Omega}_{i j} & 0 & -B_{i} K_{j}{ }^{f} \\
* & * & \Theta_{i} & B_{i} \\
* & * & * & \Sigma_{i k}
\end{array}\right) \\
Y_{i}=\left(\begin{array}{cccc}
0 & 0 & 0 & 0 \\
0 & 0 & 0 & 0 \\
0 & 0 & 0 & 0 \\
0 & -\Phi_{i} C & 0 & 0
\end{array}\right) \\
Z_{k}=\left(\begin{array}{cccc}
0 & 0 & 0 & 0 \\
0 & 0 & \Xi_{k} & 0 \\
0 & 0 & 0 & 0 \\
0 & 0 & 0 & 0
\end{array}\right)
\end{gathered}
$$




$$
P=\left(\begin{array}{ccc}
X_{1}{ }^{T} & 0 & 0 \\
0 & X_{2}^{T} & 0 \\
0 & 0 & X_{3}^{T} \\
0 & 0 & 0
\end{array}\right)
$$

and,

$$
Q=\left(\begin{array}{ccc}
\frac{3}{\mu} Q_{1} & 0 & 0 \\
0 & \frac{2}{\mu} Q_{2} & 0 \\
0 & 0 & \frac{2}{\mu} Q_{3} \\
0 & 0 & 0
\end{array}\right)
$$

where,

$$
\bar{\Omega}_{i j}=A_{i} X_{2}+X_{2} A_{i}^{T}-B_{i} W_{j}-W_{j}^{T} B_{i}^{T}
$$

and,

$$
W_{j}=K_{j} X_{2}
$$

The comparison results are illustrated in the figure 5 and the figure 6 .

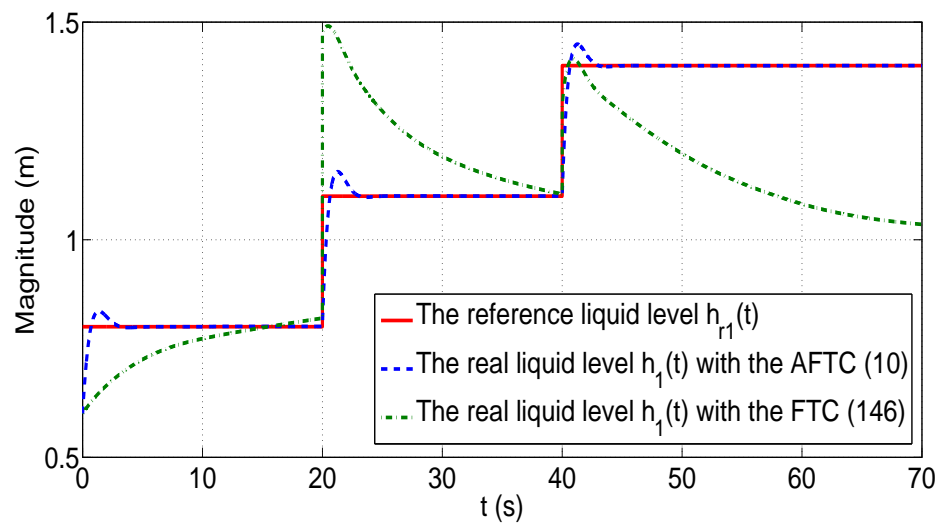

Figure 5: Comparison between the real liquid level $h_{1}(t)$ in the cases with the proposed AFTC (10) and with the FTC (143) and its reference $h_{r 1}(t)$

The figures (figure 5 and figure 6) show that the real liquid levels of the two tanks controlled by the proposed AFTC well follow their references level in spite of the presence of faults and disturbance. In addition, the fault effects are successfully compensated by the designed control law (10). These results show the effectiveness of the proposed method that allows to ensure stability, to track reference levels and to tolerate faults.

However, the FTC (143) given by the approach developed in [1], cannot keep track the reference levels. Almost from the time $40 s$, the real liquid levels $h_{1}(t)$ and $h_{2}(t)$ obtained with the FTC (143) diverge. This proves that the approach of (Aouaouda et al,) is limited for the cases of the systems affected by a certain type of faults.

The proposed APO structure presented by (6) is used to estimate the magnitudes of the faults on-line. The provided informations are necessary to reconfigure the control law. Comparing the original faults and their estimations given by the proposed observer so as to show its effectiveness. The comparison results are presented in the figure 7 and the figure 8 . Figures (figure 7 and figure 8) show that the designed APO estimates perfectly the original applied faults. 


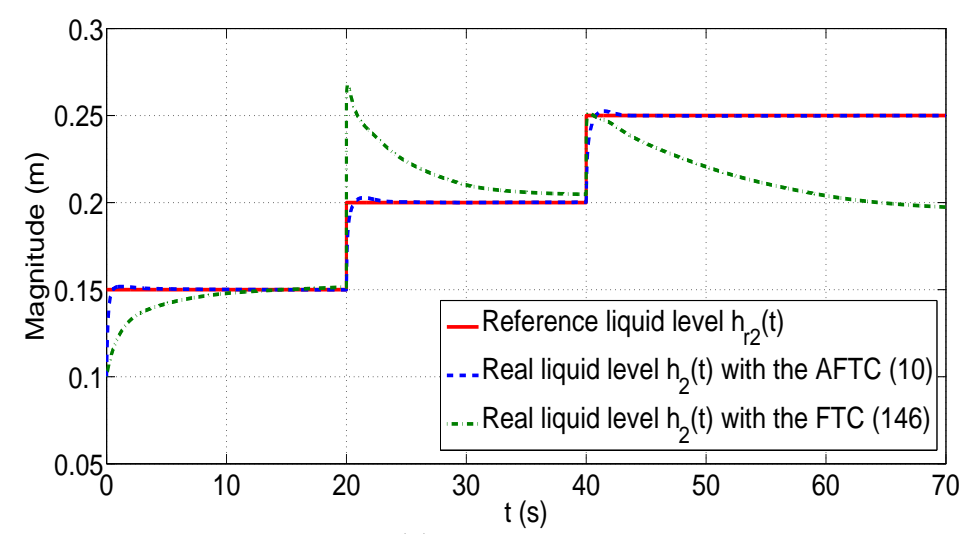

Figure 6: Comparison between the real liquid level $h_{2}(t)$ in the cases with the proposed AFTC (10) and with the FTC (143) and its reference $h_{r 2}(t)$

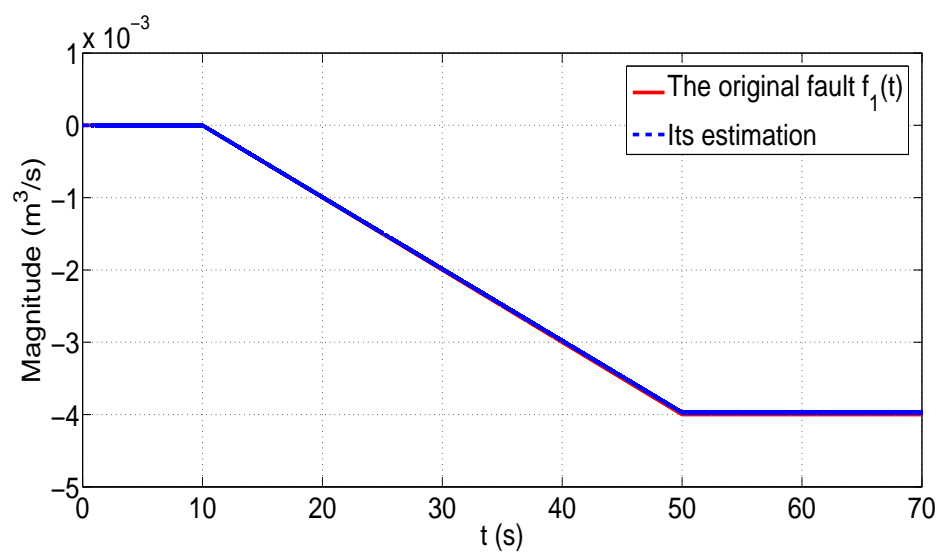

Figure 7: Comparison between the original actuator fault $f_{1}(t)$ and its estimation by using the $\mathrm{APO}$

\section{Conclusion}

In this work, the problem of model reference tracking for polytopic LPV systems with measurable gain scheduling functions has been treated. The proposed scheme consists to design a closed loop system based on an active fault tolerant controller, an Adaptive Polytopic Observer (APO) and a reference model. Such controller ensures good performances like stability, trajectory tracking, accuracy and faults compensation. The controller and the APO gains are obtained by solving a set of linear matrices inequalities (LMI). A comparaison with others previous methods underline the relevant results obtained through this new method. 


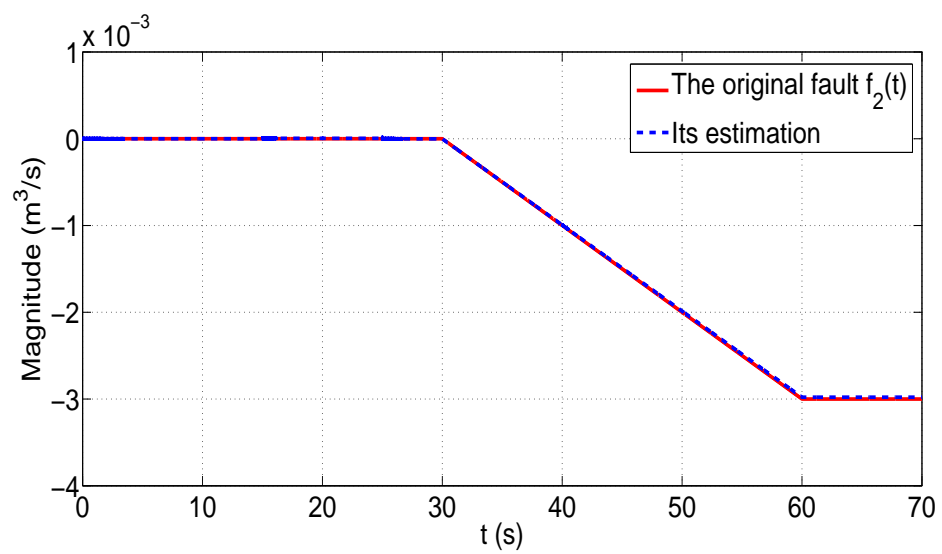

Figure 8: Comparison between the original actuator fault $f_{2}(t)$ and its estimation by using the APO

\section{References}

[1] S. Aouaouda, M. Chadli, M. T. Khadir and T. Bouarar, Robust Fault Tolerant Tracking Controller Design for Unknown Inputs TÜS Models with Unmeasurable Premise Variables. Journal of Process Control, vol.22, pp.861Û 872, 2012.

[2] S. Aouaouda and M. Chadli, Model Reference Tracking Control for Uncertain Takagi Sugeno Systems subject to Sensor Faults. The $9^{\text {th }}$ IFAC Symposium on Detection, Supervision and Safety for Technical Processes, vol.18, pp.1250-1255, 2015.

[3] C. Briat, Linear Parameter Varying and Time Delay Systems, Analysis, Observation, Filtering and Control. Advances in Delays and Dynamics, vol.3, 2015.

[4] Z.-M. Feng, G. Zhang and X.-L. Han, PID Fault Tolerant Control System Design with Multiperformance Indices Constraints. The $10^{\text {th }}$ World Congress on Intelligent Control and Automation, Beijing, China, 2012.

[5] P. Gahinet, P. Apkarian and M. Chilali, Affine Parameter-Dependent Lyapunov Functions for Real Parainetric Uncertainty. IEEE Transactions on Automatic Control, vol.41, pp.436-442, 1996.

[6] S.-M. Guo, J. S. H. Tsai, Y. C. Lin, T.-J. Tsai and C. W. Chen, Intelligent-Based PID Fault Tolerant Tracking Control for Unknown Nonlinear MIMO Systems. IEEE International Conference on Control and Automation, New Zealand, 2009.

[7] F. R. Lopez-Estrada, J. C. Ponsart, D. Theilliol and C. M. Astorga-Zaragoza, Robust $H_{-} / H_{\infty}$ Fault Detection Observer Design for Descriptor-LPV Systems with Unmeasurable Gain Scheduling Functions. International Journal of Control ,Taylor \& Francis, vol.88, pp.2380-2391, 2015.

[8] S. Montes De Oca, V. Puig, M. Witczak and L. Dziekan, Faut Tolerant Control Strategy for Actuator Faults Using LPV Techniques: Application to a Two Degree of Freedom Helicopter. International Journal of Applied Mathematics and Computer Science, vol.22, no. 1, pp.161$171,2012$. 
[9] M. Moradi and A. Fekih, Adaptive PID Sliding-Mode Fault Tolerant Control Approach for Vehicle Suspension Systems Subject to Actuator Faults. IEEE Transactions on Vehicular Technology, vol.63, no.3, pp.1041-1054, 2014.

[10] A. M. Nagy-Kiss, D. Ichalal, G. Schutz and J. Ragot, Fault Tolerant Control for Uncertain Descriptor Multimodels with Application to Wastewater Treatment Plant. American Control Conference, pp.5718-5725, Chicago (USA), 2015.

[11] H. Noura, D. Theilliol, J.-C. Ponsart and A. Chamseddine, Fault-Tolerant Control Systems: Design and Practical Applications. Springer, 2009.

[12] M. Rodrigues, H. Hamdi, N. BenHadj Braiek, D. Theilliol, Observer based Fault Tolerant Control Design for a Class of LPV Descriptor Systems. Journal of The Franklin Institute, pp.1-20, 2014.

[13] M. Rodrigues, H. Hamdi, D. Theilliol, C. Mechmeche and N. BenHadj Braiek, Actuator Faut Estimation based Adaptive Polytopic Observer for a Class of LPV Descriptor Systems. International Journal of Robust and Nonlinear Control, pp.1-15, 2014.

[14] D. Rotondo,F. R. Lopez-Estrada, F. Nejjari, J. C. Ponsart, D. Theilliol and V. Puig, Actuator Multiplicative Fault Estimation in Discrete-Time LPV Systems Using Switched Observers. Journal of the Franklin Institute, Elsevier, vol.353 (13), pp.3176-3191, 2016.

[15] D. Rotondo, F. Nejjari and V. Puig, 2013. Quasi-LPV modeling, identification and control of a twin rotor MIMO system. Control Engineering Practice, vol.21 (6), pp.829Ü846, 2013.

[16] D. Rotondo, F. Nejjari and V. Puig, A Virtual Actuator and Sensor Approach for Fault Tolerant Control of LPV Systems. Journal of Process Control, vol.24, pp.203-222, 2014.

[17] D. Rotondo, V. Puig, F. Nejjari, and J. Romera, A fault-hiding approach for the switching quasi-LPV fault-tolerant control of a four-wheeled omnidirectional mobile robot. IEEE Transactions on Industrial Electronics, vol.62 (6), pp.3932Û3944, 2015.

[18] D. Rotondo, V. Reppa, V. Puig and F. Nejjari, Adaptive Observer for Switching Linear Parameter-Varying (LPV) Systems. The $19^{\text {th }}$ International Federation of Automatic Control World Congress, vol.47 (3), pp.1471-1476, 2014.

[19] Y. Zhang and J. Jiang, Bibliographical Review on Reconfigurable Fault Tolerant Control Systems. Annual Reviews in Control, vol.32, pp.229-252, 2008. 